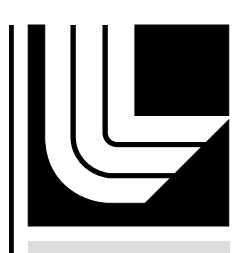

LAWRENCE LIVERMORE NATIONAL LABORATORY

X-ray Diffraction Diagnostic Design for the National Ignition Facility

M. Ahmed, A. House, R. F. Smith, Z. Lamb, D. W. Swift

July 31,2013

SPIE Conference

San Diego, CA, United States

August 25, 2013 through August 29, 2013 
This document was prepared as an account of work sponsored by an agency of the United States government. Neither the United States government nor Lawrence Livermore National Security, LLC, nor any of their employees makes any warranty, expressed or implied, or assumes any legal liability or responsibility for the accuracy, completeness, or usefulness of any information, apparatus, product, or process disclosed, or represents that its use would not infringe privately owned rights. Reference herein to any specific commercial product, process, or service by trade name, trademark, manufacturer, or otherwise does not necessarily constitute or imply its endorsement, recommendation, or favoring by the United States government or Lawrence Livermore National Security, LLC. The views and opinions of authors expressed herein do not necessarily state or reflect those of the United States government or Lawrence Livermore National Security, LLC, and shall not be used for advertising or product endorsement purposes. 


\title{
$X$-ray diffraction diagnostic design for the National Ignition Facility
}

\author{
Maryum F. Ahmed, Allen House, R. F. Smith, Zachary S. Lamb, David W. Swift \\ Lawrence Livermore National Laboratory, 7000 East Avenue, Livermore, CA 94550
}

\begin{abstract}
This paper describes the design considerations for Target Diffraction In-Situ (TARDIS), an x-ray diffraction diagnostic at the National Ignition Facility. A crystal sample is ramp-compressed to peak pressures between 10 and 30 Mbar and, during a pressure hold period, is probed with quasi-monochromatic x-rays emanating from a backlighter source foil. The crystal spectrography diffraction lines are recorded onto image plates. The crystal sample, filter, and image plates are packaged into one assembly, allowing for accurate and repeatable target to image plate registration. Unconverted laser light impinges upon the device, generating debris, the effects of which have been mitigated. Dimpled blast shields, high strength steel alloy, and high-z tungsten are used to shield and protect the image plates. A tapered opening was designed to provide adequate thickness of shielding materials without blocking the drive beams or $\mathrm{x}$-ray source from reaching the crystal target. The high strength steel unit serves as a mount for the crystal target and x-ray source foil. A tungsten body contains the imaging components. Inside this sub-assembly, there are three image plates: a 160 degree field of view curved plate directly opposite the target opening and two flat plates for the top and bottom. A polycarbonate frame, coated with the appropriate filter material and embedded with registration features for image plate location, is inserted into the diagnostic body. The target assembly is metrologized and then the diagnostic assembly is attached.
\end{abstract}

Keywords: X-ray, diffraction, spectrography, NIF, Target Diffraction In-Situ (TARDIS), laser

\section{INTRODUCTION}

Target Diffraction In-Situ (TARDIS) is an x-ray diffraction diagnostic that will be used for in-situ characterization of crystal structures and textures of materials ramp compressed to high levels of compression.

TARDIS will be used at the National Ignition Facility (NIF), located at Lawrence Livermore National Laboratory. NIF is the highest energy laser ever constructed ${ }^{1}$. It has been used to compress Tantalum to over $10 \mathrm{Mbar}$, and diamond to 50 Mbar, recreating the most extreme planetary conditions, in excess of all planets in our solar system, except Jupiter ${ }^{2}$.

The facility includes 192 lasers, assembled in groups of 4, or "quads". Up to 6 laser quads can be directed at the TARDIS crystal sample, compressing the target to pressures up to 30Mbar. Up to 4 quads can be used to illuminate a backlighter source foil, which probes the crystal with $\mathrm{He}-\alpha \mathrm{keV}$ x-rays, projecting crystal diffraction lines inside the TARDIS housing. The lines are recorded onto image plates, allowing for characterization of the crystal structure of the compressed sample.

\subsection{Overview}

TARDIS is the first project to include a NIF target and diagnostic on a single, integrated platform. The system is separated into two units, the target assembly and the diagnostic assembly, shown in Figure 1. The target assembly contains the main crystal sample, the backlighter foil, alignment fibers and shielding components. The shielding components are used to mitigate the effects of unconverted laser light impinging upon the TARDIS body. The diagnostic assembly contains the image plates, filters and additional high-Z tungsten shielding.

The diagnostic body seats inside of the target body, as shown in the cutaway view in Figure 2. A pair of precision alignment pins guides the diagnostic body into position in the $\mathrm{Y}$ and $\mathrm{Z}$ directions. Four standoffs control the $\mathrm{X}$ position. Two fasteners, one on each side of the assembly, clamp the diagnostic body to the target body. Belleville disc springs under these two fastener heads allow compliance in the joints. This allows displacement of the diagnostic body with respect to the target at the time of impact, reducing stresses throughout the structural components. 


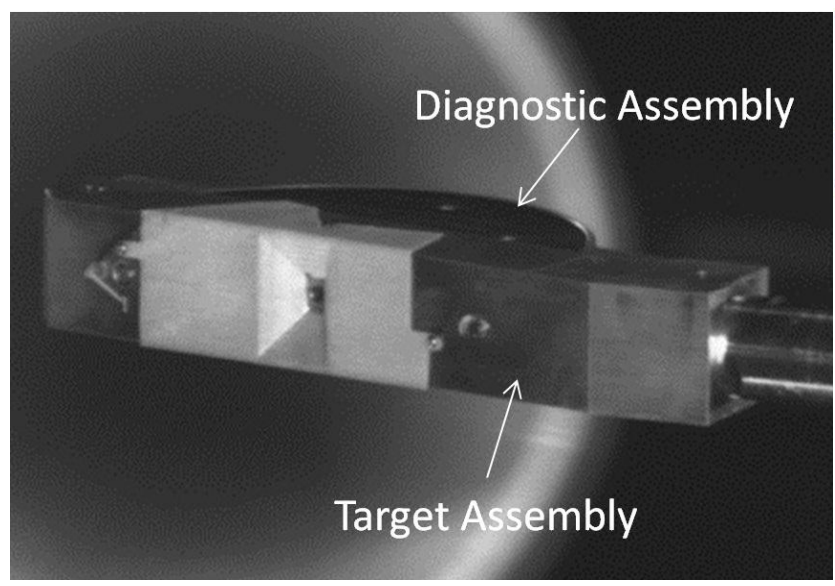

(a.)

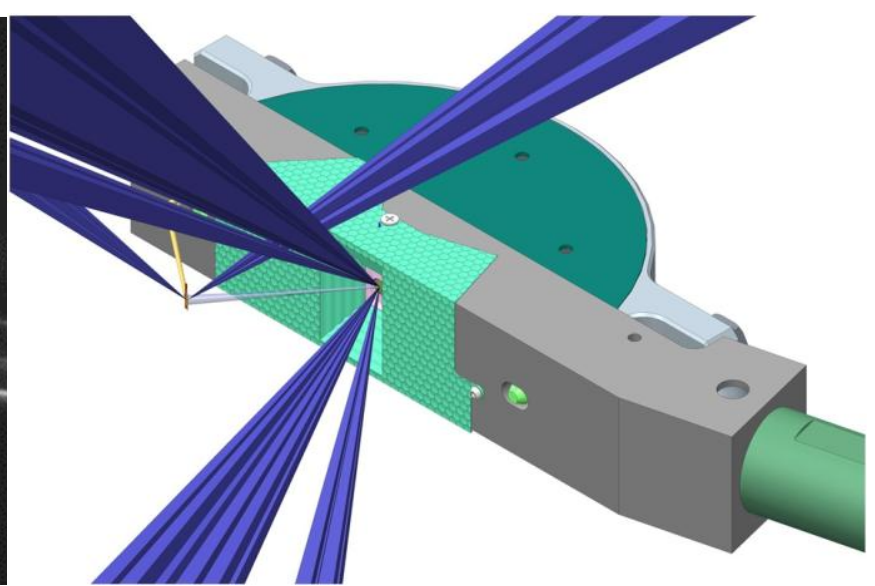

(b.)

Figure 1. (a.) TARDIS mounted in the target chamber. (b.) Depiction of TARDIS with 6 quads aimed at crystal sample and

2 quads aimed at backlighter

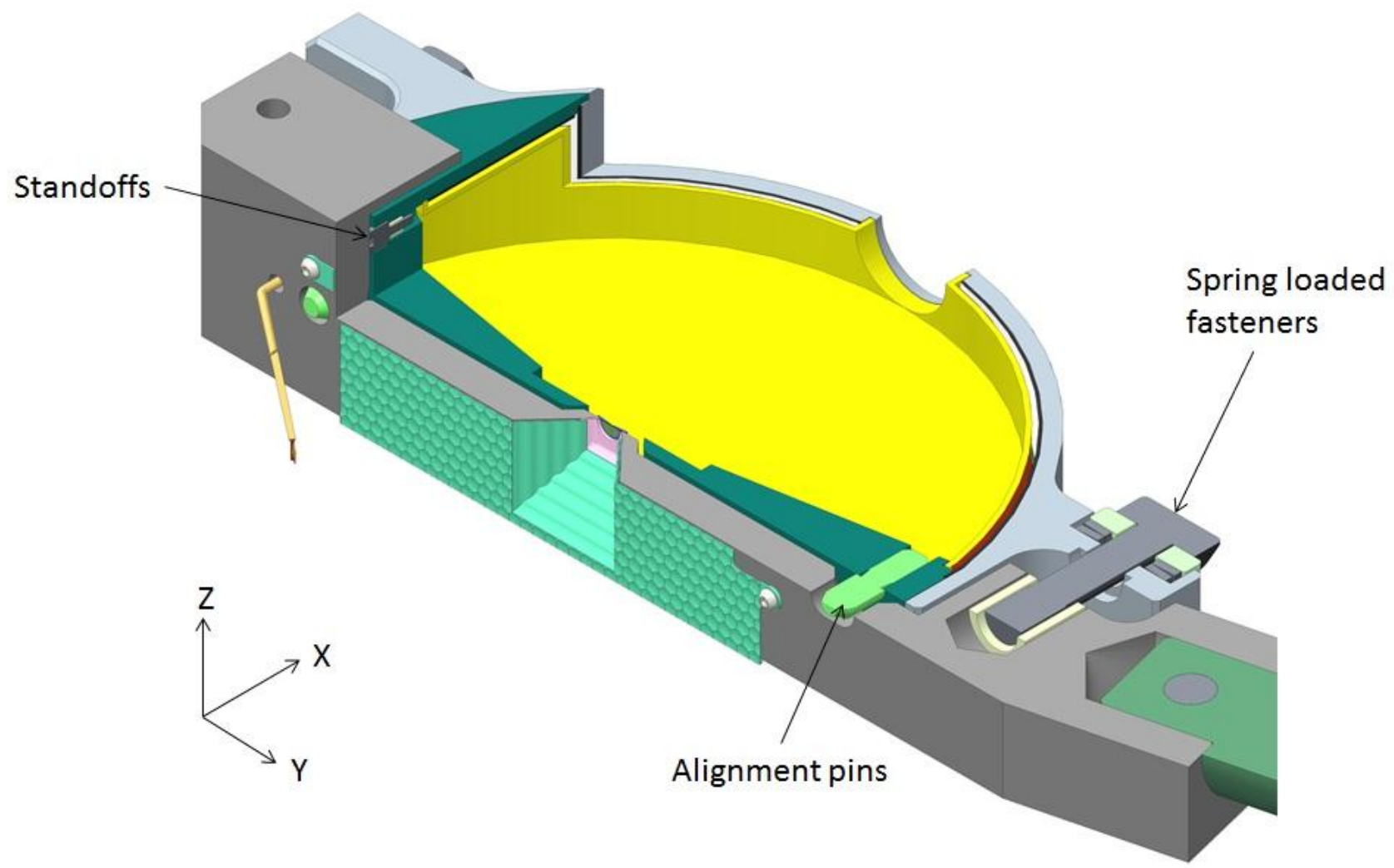

Figure 2. Cutaway depiction of TARDIS assembly. Alignment pins control the $\mathrm{Y}$ and $\mathrm{Z}$ positioning of the diagnostic assembly. Standoffs control the $\mathrm{X}$ position. Spring loaded fasteners clamp the bodies together.

\subsection{Debris mitigation}

Unconverted $1 \omega$ and $2 \omega$ laser light impinges upon the device, with a potential to create debris which poses a risk of damaging both TARDIS and other systems within the NIF target chamber. 


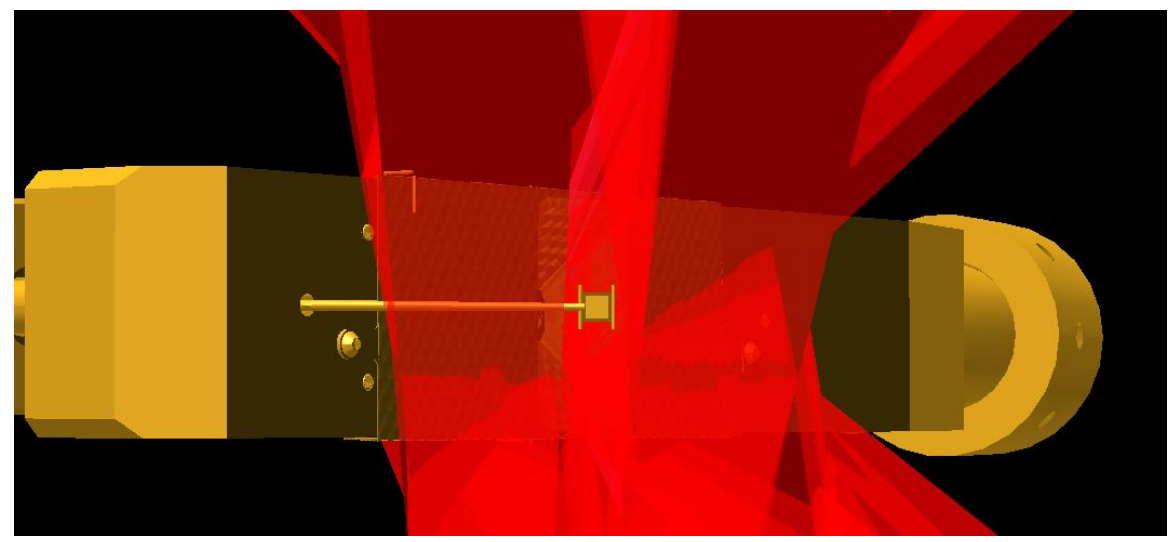

Figure 3. Simulation of TARDIS blast shields bathed in unconverted light.

The laser to material interaction was analyzed using the laser fusion simulation software, LASNEX, generating a pressure vs. time history at the surface of the TARDIS blast shields. The results were used for finite element analysis of the major structural components of the TARDIS assembly.

It was determined that the combination of $100 \mu \mathrm{m}$ minimum thickness dimpled plastic blast shield, $300 \mu \mathrm{m}$ vacuum gap, $2 \mathrm{~mm}$ thick high strength steel alloy, and $2 \mathrm{~mm}$ thick tungsten alloy would result in no material spalling inside the TARDIS diagnostic body, thus protecting the image plates from damage and minimizing potential loss of data.

Detailed radiation-hydrodynamic simulations were performed to predict the mass and trajectory of any debris resulting from the laser drive beams impinging on the sample. The results indicate that any debris escaping the TARDIS housing is minimal, and that any risk posed to the image plates and nearby sensors is within acceptable limits.

\section{DIAGNOSTIC ASSEMBLY}

The diagnostic assembly has a tungsten body as the main structural component, depicted in Figure 4. The image plates and filter are housed inside this main body, with a curved back plate enclosing the assembly. The alignment pins and standoffs, used for positioning the diagnostic unit are fixed to the tungsten body. The back plate, curved image plate and filter have apertures for viewing of the target by the Velocity Interferometer System for Any Reflector (VISAR) diagnostic ${ }^{3}$.

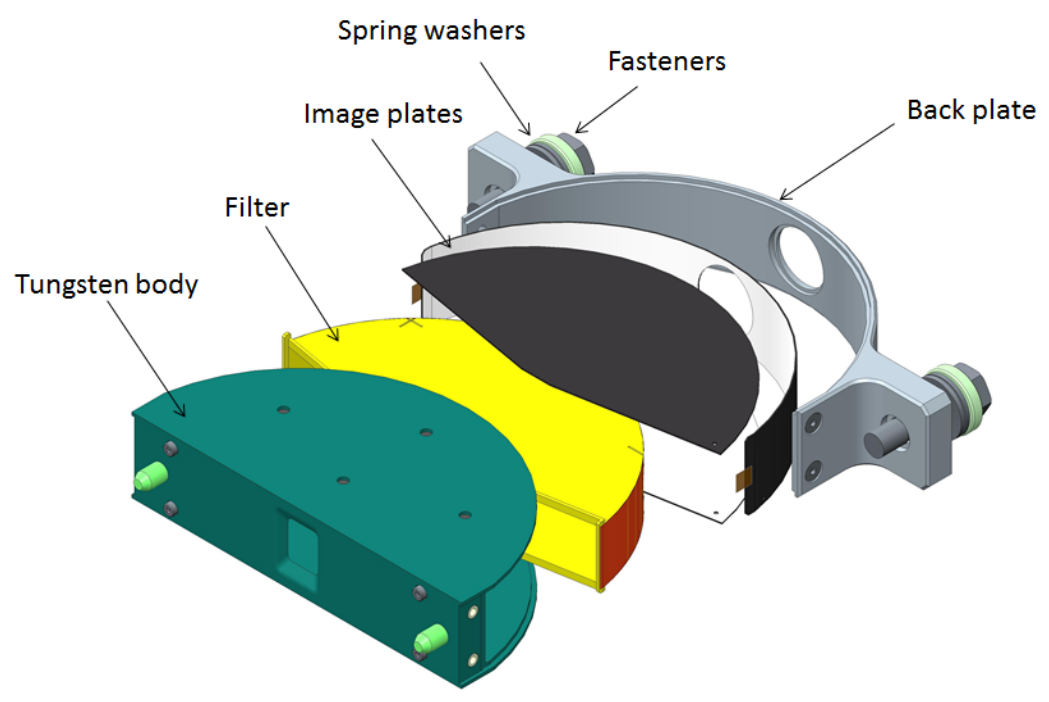

Figure 4. The TARDIS diagnostic assembly: tungsten body, filter assembly, image plates, back plate, spring washers and fasteners.. 


\subsection{Image plates}

The diagnostic body holds three reusable phosphor screen image plates, as depicted in Figure 4. The image plates display greater linearity with dose over a wider dynamic range than film emulsion and do not require chemical development, making them ideal for use in x-ray spectrometry applications ${ }^{4}$.

The top and bottom plates are flat and held in place by small disc magnets embedded in the tungsten body. The back plate, opposite the crystal sample is curved by aligning the edges between the tungsten body and the back plate. It is positioned with the center of curvature coincident with the target pinhole. This ensures near normal incidence of x-rays on the image plate. The required data collection angle for TARDIS was analyzed based on sample and pinhole properties and determined to be $+/-76^{\circ}$. The positioning of the image plates relative to the target is critical for the quality of the data. The back surfaces upon which the image plates are held are machined to tight tolerances to maintain accurate distances from the image plates to the target.

\subsection{Filters}

The TARDIS filters are built up in several steps. The main filter base is a rapid prototyped, polycarbonate frame. Thin, $250 \mu \mathrm{m}$ tungsten fiducial wires are fixed inside grooves built into the base. The tungsten blocks imaging onto the image plates, recording fiducial marks. The marks are used for accurately correlating the data between the 3 plates.

Separately, thin polyimide sheets are coated with the filter material, such as germanium, zirconium, zinc or copper. The coated sheets are fixed to the frame using a colorless epoxy. The thicknesses of the materials are varied by experiment and can be applied to some or all of the three filter faces, depending on individual experiment requirements. A black opaque polyimide sheet is fixed to each of the three exterior surfaces, also using colorless epoxy. A covered TARDIS filter is shown in Figure 5. Finally, any uncovered polycarbonate edges are painted with an opaque graphite coating, to protect the image plates from external light sources.

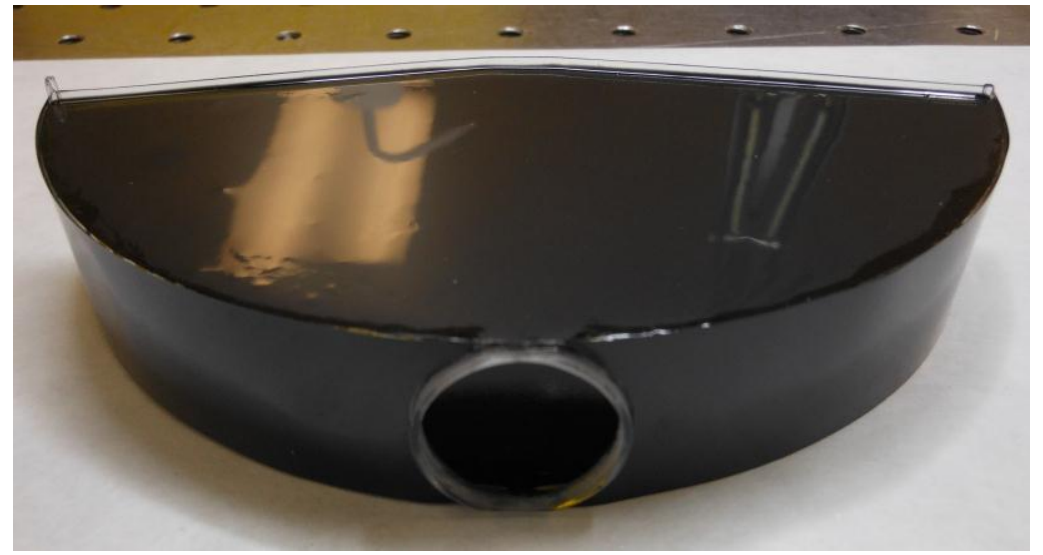

Figure 5. TARDIS filter consisting of polycarbonate frame, coated polyimide sheets, and black polyimide sheets. Graphite coating has not yet been applied to clear polycarbonate edges.

\section{TARGET ASSEMBLY}

The target assembly and its components are depicted in Figure 6. The assembly has a high strength steel alloy body as the main structural component on which the crystal sample and backlighter foil are mounted. The assembly holds the dimpled blast shields which are covered by unconverted laser light during the shot. The diagnostic assembly is mounted into a pocket on the back of the target assembly, where it is protected from the unconverted light. 


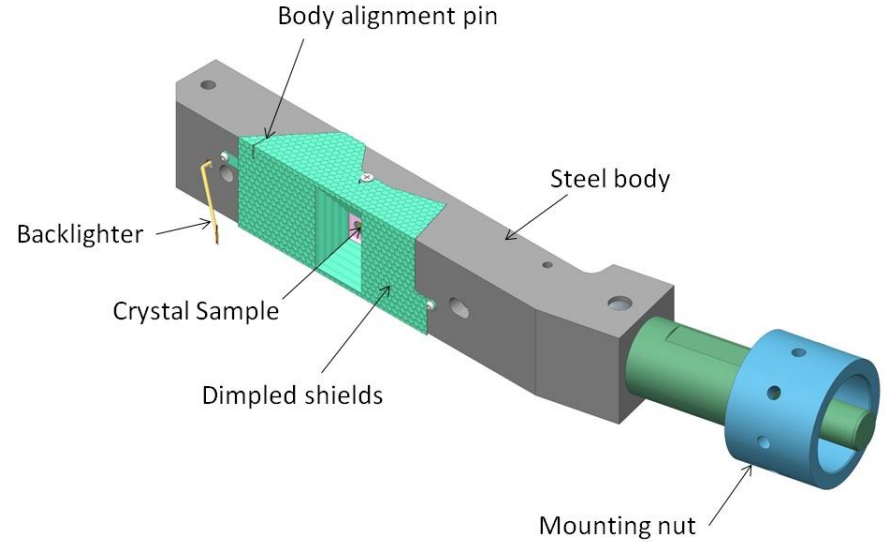

(a.)

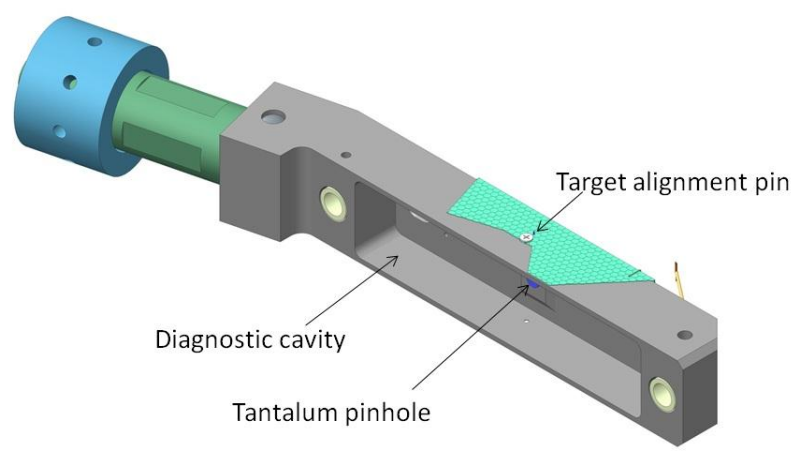

(b.)

Figure 6. The TARDIS target assembly. (a.) Front side of target assembly. NIF beams impact the crystal sample from the front side. (b.) Back side of target assembly. Diagnostic assembly mounts to back side.

The TARDIS is supported and inserted into the target chamber by the Target Positioner (TARPOS), which holds noncryogenic NIF targets. The steel body also serves to mount the TARDIS to the TARPOS, via the arm extension and mounting nut. A typical NIF target weighs only a few grams, whereas the TARDIS weighs just under $2.5 \mathrm{~kg}$ and transfers high loads due to the impingement of unconverted light. The TARPOS bolted connections were analyzed using the results of the TARDIS finite element analysis and modified to withstand the higher loads.

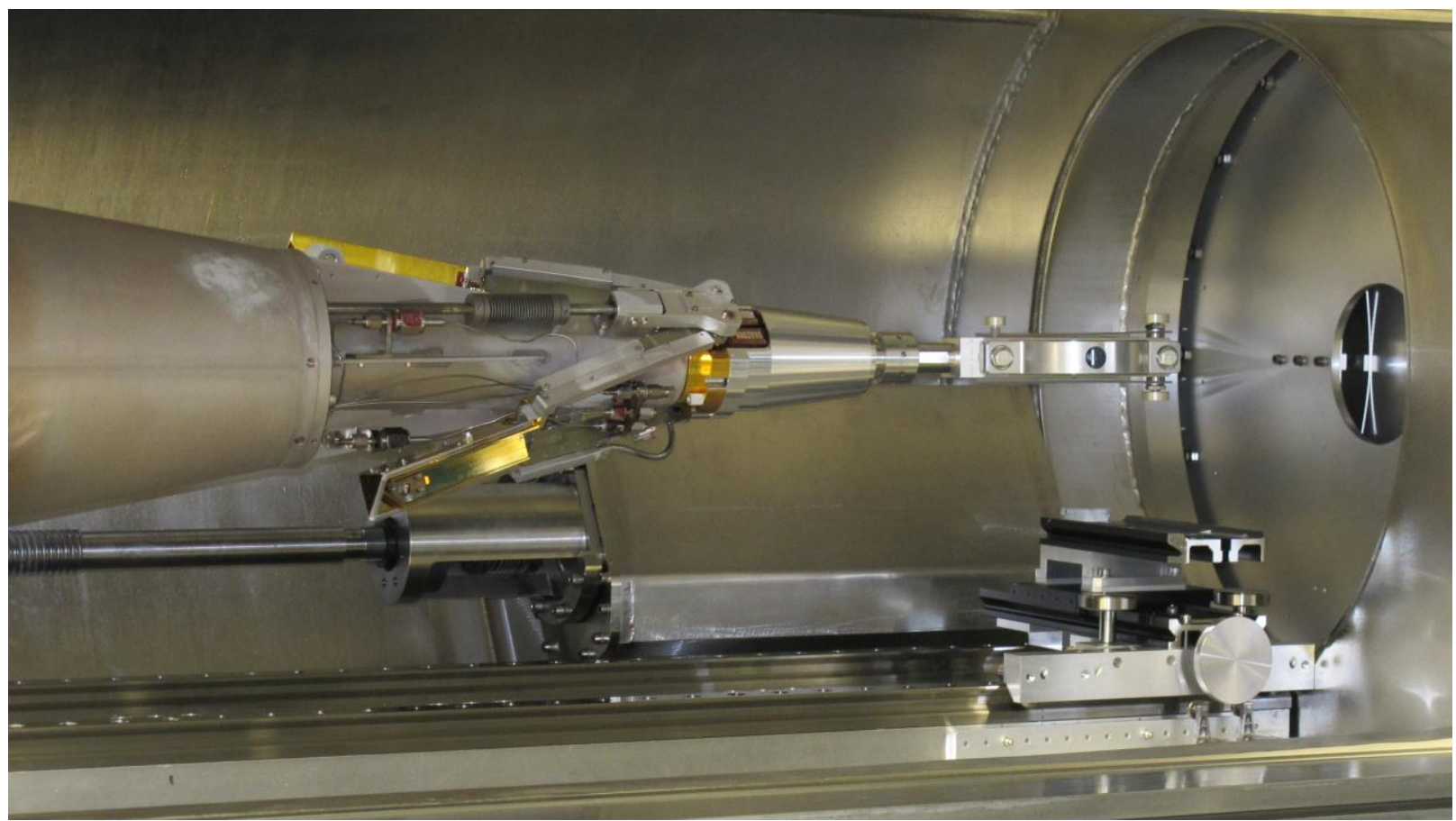

Figure 7. TARDIS with protective cover, mounted on Target Positioner (TARPOS). 


\subsection{Dimpled shield}

Dimpled blast shields, made of $\mathrm{CH}$ plastic polymer or $\mathrm{CH}$ coated aluminum are fixed to the front face of the steel body. The dimpled surface prevents retroreflection of laser light so that it does not propagate back through the laser chain. These shields are the assembly's first line of defense against the unconverted laser light, and are intended to be vaporized, with little or no material remaining after the experiment.

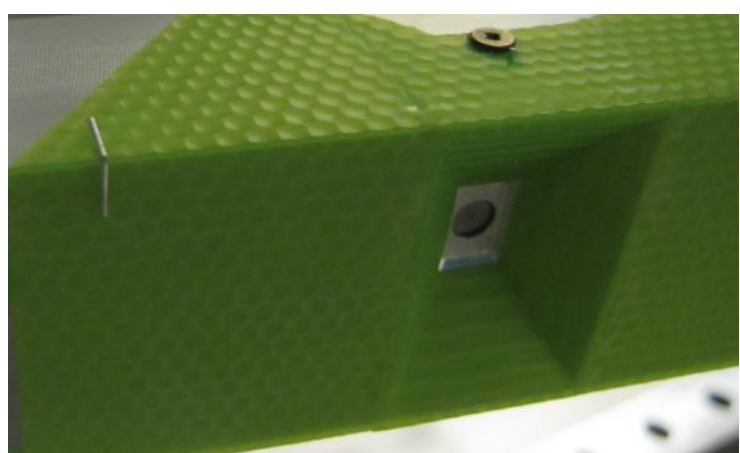

(a.)

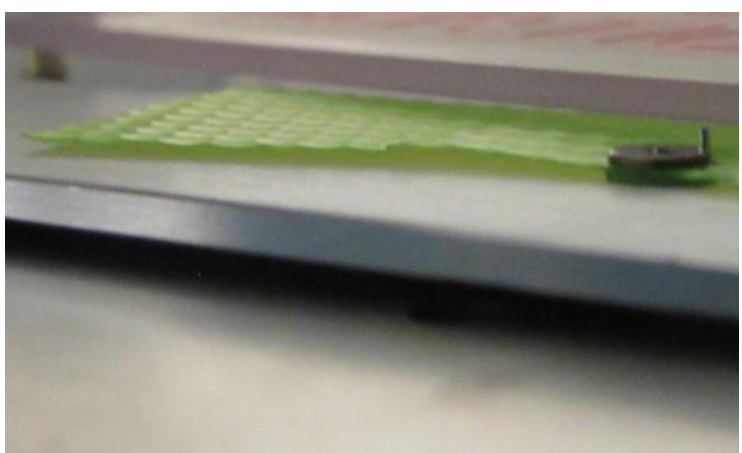

(b.)

Figure 8. (a.) Front plastic blast shield with target opening and L-shaped alignment fibers. (b.) Top plastic blast shield showing $300 \mathrm{~mm}$ vacuum gap and upper alignment fiber.

\subsection{Tapered opening}

The requirement for data collection at angles of $+/-76^{\circ}$, combined with the shielding requirements, described in section 1.2, drove the tapered opening design, shown in Figure 8(a.) and Figure 9. The opening allows a clear path for $3 \omega$ laser light to reach the crystal sample, while blocking the path of the unconverted laser light. The pressure wave formed from the unconverted light impinging upon the body travels normal to the surface of the body, allowing adequate space for required shielding material in the path of the pressure wave. The protrusion of the target into the diagnostic body allows for a clear path from sample pinhole to curved image plate of $+/-80^{\circ}$, meeting the requirement for data collection.

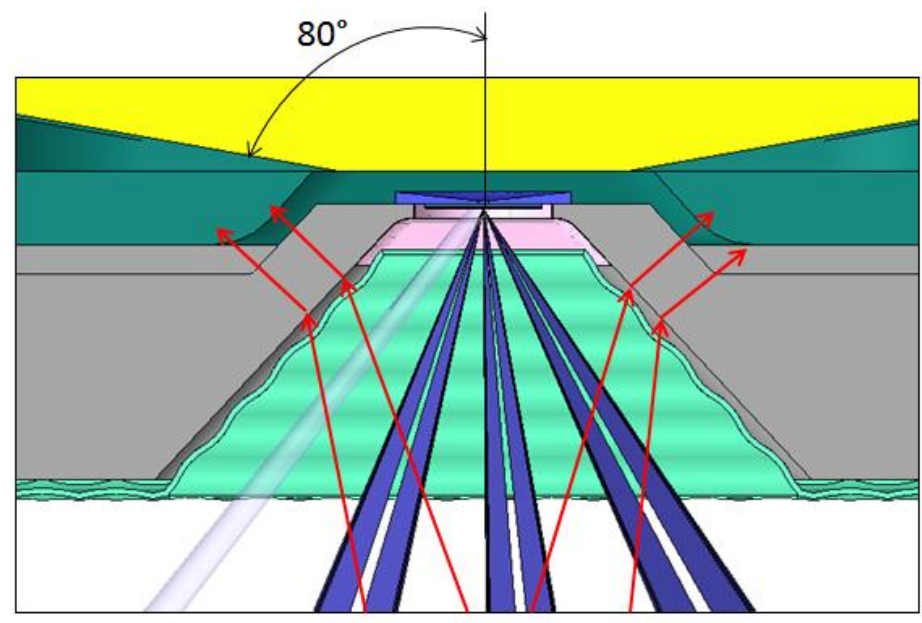

Figure 9. Cross section of tapered opening with $3 \omega$ laser light and x-rays directed at crystal sample. Arrows represent direction of unconverted laser light and corresponding pressure wave.

\subsection{Crystal sample}

The crystal sample is a $4 \mathrm{~mm}$ diameter disc composed of single crystal diamond layers, and other materials, bonded together using thin, under $3 \mu \mathrm{m}$ thick adhesive. The thicknesses of the layers vary by experiment and are measured to within $1 \mu \mathrm{m}$. The sample is fixed to the center, front surface of a tantalum pinhole disc, depicted in figure 9. The 
tantalum contains a $400 \mu \mathrm{m}$ diameter pinhole for initial shots, but may vary based on specific experiment requirements. The outer front surface of the tantalum is fixed to the back surface of the protrusion on the steel body, positioning the crystal sample inside the opening. This configuration allows the pinhole location to stay the same, regardless of sample thickness.

\subsection{Backlighter}

The backlighter, shown in Figure 10, is a thin foil material, which will be impinged upon by NIF beams, creating the quasi-monochromatic He- $\alpha$ x-ray source which will probe the crystal sample at the time of maximum compression. An experiment specific material, such as germanium or zirconium is directly coated to a thin substrate of either graphite or polyimide. The backlighter foil and alignment fiber are supported by a rapid prototype polymer stalk, mounted to the front face of the TARDIS.

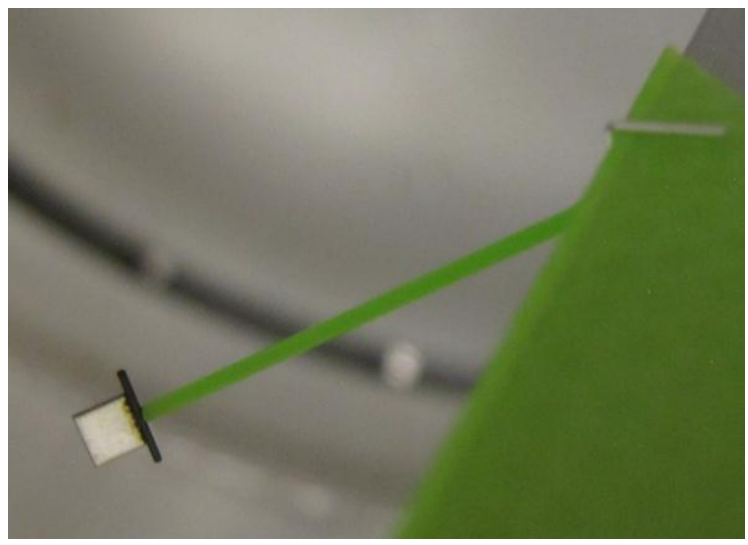

Figure 10. TARDIS backlighter foil and alignment fiber, supported by polymer stalk.

\subsection{Alignment fibers}

During insertion into the target chamber, TARDIS is aligned using the Target Alignment Sensor (TAS). TAS uses four alignment fibers to position TARDIS. As the TARDIS is inserted into the TAS jaws, the alignment cameras will first see the L-shaped, corner fiber, shown in Figure 8(a). This gives operators a "marker" to look for before full insertion. An upper alignment fiber shown on the far right side in Figure 8(b), and a similar lower alignment fiber are positioned directly above and below the sample's front surface and any errors in the positioning of these fibers are measured and known ahead of time. These are used for fine tuning the position of the crystal sample and for laser pointing. A long alignment fiber, shown in Figure 10, is fixed to the backlighter stalk, and also used for laser pointing.

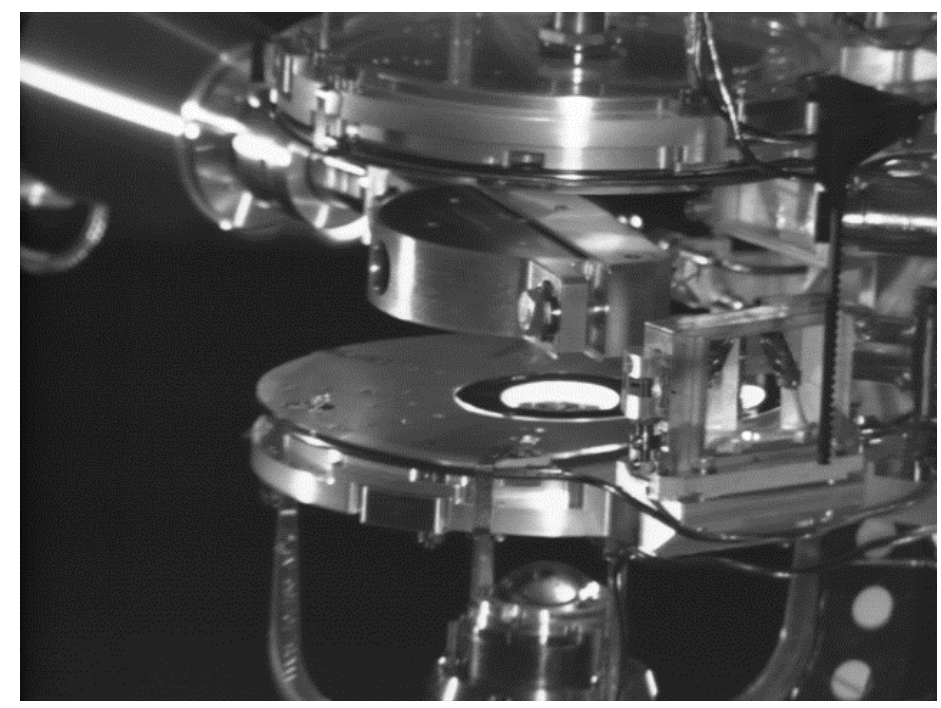

Figure 11. TARDIS, mounted on the TARPOS, inserted into the Target Alignment Sensor (TAS) in the NIF target chamber. 


\section{CONCLUSION}

The TARDIS assembly has gone through initial dry runs including assembly of the diagnostic and target units and installation to the TARPOS (Target Positioner) arm. Positioning and alignment in the NIF target chamber, using the TAS were successfully completed. This test was of particular importance, given the size of the TARDIS compared to any other system that has previously been aligned using these systems. As of the completion of this paper, the first TARDIS experiments are still pending.

Going forward, a modified hemispherical shaped filter may be developed. This would allow for uniform filtering of diffraction lines, increasing the quality of the resulting data. Preliminary tests of hemispherical filters have been tested on the OMEGA laser at the Laboratory for Laser Energetics, with promising results.

Utilizing the precise laser pulse shaping on NIF we can compress our samples to high pressures and low temperatures thereby keeping the material in the solid state at extreme levels of compressions found within the cores of giant planets ( 10's of Mbar). The TARDIS x-ray diffraction diagnostic described here will allow researchers to quantify the change in crystal structure as a function of applied pressure data which is expected to have profound impact on our understanding of planetary evolution.

\section{REFERENCES}

[1] E. I. Moses, R. N. Boyd, B. A. Remington, C. J. Keane, and R. Al-Ayat, "The National Ignition Facility: Ushering in a new age for high energy density science," AIP Physics of Plasmas 16, 041006 (2009)

[2] R. F. Smith, J. H. Eggert, D. G. Braun, J. R. Patterson, R. E. Rudd, R. Jeanloz, T. S. Duffy, J. Biener, A. E. Lazicki, A.V. Hamza, J. Wang, T. Braun, L. X. Benedict, P. M. Celliers, G. W. Collins, "Diamond at 5TPa", manuscript in preparation.

[3] R. M. Malone, B. C. Frogget, M. I. Kaufman, T. W. Tunnell, R. L. Guyton, I. P. Reinbachs, P. W. Watts, J. R. Celeste, P. M. Celliers, T. L. Lee, B. J. MacGowan, E. W. Ng, R. B. Robinson, and L. G. Seppala, " Overview of the Line-Imaging VISAR Diagnostic at the National Ignition Facility (NIF)," in International Optical Design, Technical Digest (CD) (Optical Society of America, 2006), paper ThA5.

[4] B. R. Maddox, H.-S. Park, B. A. Remington, N. Izumi, S. Chen, C. Chen, G. Kimminau, Z. A. Ali, M. J. Haugh, Q. Ma, "Characterization of the FLA7000 Image Plate System for Quantitative X-ray Detection Applications," AIP Review of Scientific Instruments, 2009

This work performed under the auspices of the U.S. Department of Energy by Lawrence Livermore National Laboratory under Contract DE-AC52-07NA27344. 